\title{
REMARKS ON THE CARDINALITY OF COMPACT SPACES AND THEIR LINDELÖF SUBSPACES
}

\author{
A. HAJNAL AND I. JUHÁSZ
}

\begin{abstract}
Several applications of the $\check{C}$ ech-Pospišil theorem are given; one of them states (under $\mathrm{CH}$ ) that every uncountable compact space has a Lindelö subspace of cardinality $\omega_{1}$.
\end{abstract}

Notation and definitions are the same as in [1], except that a $G_{\kappa}$ set means a set which is the intersection of $\leqslant \kappa$ open sets. Thus if $\kappa=\omega_{\xi}$, then $G_{\kappa}$ is the same as $G_{\delta, \xi}$ in [1].

Let us recall at the outset the old theorem of Čech and Pospišil which states that if $X$ is compact $T_{2}$ and $\chi(p, X) \geqslant \kappa \geqslant \omega$ hold for each $p \in X$, then $|X| \geqslant 2^{\kappa}$. In [1] and [2] it is (incorrectly) stated that this theorem remains valid if we only require $X$ to be a $G_{\kappa}$ subset of some compact $T_{2}$ space. This is not true; however, it turns out that if we replace $G_{\kappa}$ with $G_{\lambda}$ for some $\lambda<\kappa$, then a simple argument can reduce it to the Cech-Pospišil theorem.

Proposition 1. If $X \subset Z$ is a (nonempty) $G_{\lambda}$-subset with $\lambda<\kappa$, where $Z$ is compact Hausdorff, and $\chi(p, X) \geqslant \kappa$ for each $p \in X$, then $|X| \geqslant 2^{\kappa}$.

Proof. It is known (cf. [1, 2.11]) that $X$ contains a nonempty subset $Y$ which is a closed $G_{\lambda}$-subset of $Z$. Thus $Y$ is compact and we claim that $\chi(p, Y) \geqslant \kappa$ for each $p \in Y$. Indeed, since $\psi(p, Y)=\chi(p, Y)$, otherwise we would have $\psi(p, Z)=\chi(p, Z)<\kappa$, considering that $Y$ is a $G_{\lambda}$-subset of $Z$, contradicting that $\kappa \leqslant \chi(p, X) \leqslant \chi(p, Z)$. Hence we have $2^{\kappa} \leqslant|Y| \leqslant|X|$.

The following result is not proved directly from the Čech-Pospišil theorem but from its proof. It seems to be new and will be crucial in the proof of Theorem 1 .

Proposition 2. If $X$ is a compact Hausdorff space with $\chi(p, X) \geqslant \kappa$ for all $p \in X$, then there exists a subset $C \subset X$ with $|C| \leqslant 2^{\kappa}=\sum\left\{2^{\lambda}: \lambda<\kappa\right\}$ such that $|\bar{C}| \geqslant 2^{\kappa}$.

Proof. The basic idea in all known proofs of the Cech-Pospišil theorem is the construction of a certain kind of ramification system of closed sets. To be more precise, for every $0-1$ sequence $\varepsilon$ of length $\nu \leqslant \kappa$ one defines a nonempty closed set $X_{\varepsilon} \subset X$ such that

(i) if $\nu$ is a limit ordinal, then $X_{\varepsilon}=\cap\left\{X_{\varepsilon \uparrow \mu}: \mu<\nu\right\}$;

(ii) $X_{\hat{\varepsilon} \hat{0}}, X_{\hat{\varepsilon} 1} \subset X_{\varepsilon}$ and $X_{\hat{\varepsilon} 0} \cap X_{\hat{\varepsilon} 1}=\varnothing$.

Let $S_{\nu}$ denote the set of all $0-1$ sequences of length $\nu$. For every $\nu<\kappa$, $\varepsilon \in S_{\nu}$ and $i<2$, the set $X_{\varepsilon} \backslash X_{\widehat{\varepsilon} i}$ is nonempty; hence we can choose for each

Received by the editors March 13, 1975.

AMS (MOS) subject classifications (1970). Primary 54A25, 54D30.

(1) American Mathematical Society 1976 
such $\varepsilon$ and $i$ a point $p_{\varepsilon, i} \in X_{\varepsilon} \backslash X_{\widehat{\varepsilon} i}$. Now put

$$
C=\left\{p_{\varepsilon, i}: \varepsilon \in \cup\left\{S_{\nu}: \nu<\kappa\right\} \& i \in 2\right\} .
$$

It is obvious that $|C| \leqslant 2^{\kappa}$. Next we show that for each $\eta \in S_{\kappa}$ we have $X_{\eta} \cap \bar{C} \neq \varnothing$; this will obviously imply $|\bar{C}| \geqslant 2^{\kappa}$. To see this, let

$$
C_{\eta}=\left\{p_{\eta \uparrow \nu, \eta(\nu)}: \nu<\kappa\right\} \subset C .
$$

(For simplicity we write $p_{\eta \uparrow \nu, \eta(\nu)}=p^{(\nu)}$.) Then $\mu<\nu<\kappa$ implies $p^{(\mu)} \neq p^{(\nu)}$, hence $\left|C_{\eta}\right|=\kappa$. Since $X$ is compact, $C_{\eta}$ must have a complete accumulation point in $X$, and we claim that any such point must belong to $X_{\eta}$. This will imply $\bar{C} \cap X_{\eta} \supset \bar{C}_{\eta} \cap X_{\eta} \neq \varnothing$. Indeed, let $q \in X \backslash X_{\eta}$ arbitrary. Then there is a $\nu<\kappa$ such that $q \in X \backslash X_{\eta \uparrow \nu}$, but obviously, this latter set is an open neighbourhood of $q$, whose intersection with $C_{\eta}$ is $\left\{p^{(\mu)}: \mu<\nu\right\}$, hence of cardinality less than $\kappa$. This completes the proof.

The next result was obtained while trying to solve the following problem: Is it true that a Lindelöf space of cardinality $\omega_{2}$ must contain a Lindelöf subspace of cardinality $\omega_{1}$ ? ( $\mathrm{GCH}$ is assumed.) A natural thing was to try it for compact spaces first.

THEOREM 1. Assume the CH (i.e. $2^{\omega}=\omega_{1}$ ). Then every uncountable compact space has a Lindelöf subspace of cardinality $\omega_{1}$.

Before we can start the actual proof, we state a lemma, which is interesting in itself.

Lemma. If a space $X$ has a point $p \in X$ with $\psi(p, X)=\chi(p, X)=\omega_{1}$, then $X$ contains a Lindelöf subspace of cardinality $\omega_{1}$.

Proof. Let $\left\{U_{\nu}: \nu<\omega_{1}\right\}$ be a basis of neighbourhoods for $p$ in $X$. We define points $p_{\nu}$ for $\nu<\omega_{1}$ by transfinite induction, as follows. Suppose that $\nu<\omega_{1}$, and for each $\mu<\nu$ the point $p_{\mu}$ has already been defined.

Then we choose $p_{\nu}$ in $\cap\left\{U_{\mu}: \mu \leqslant \nu\right\} \backslash\left(\left\{p_{\mu}: \mu<\nu\right\} \cup\{p\}\right)$, which is possible by $\psi(p, X)=\omega_{1}$.

Now let us put $S=\left\{p_{\nu}: \nu<\omega_{1}\right\} \cup\{p\}$ and let $G$ be any neighbourhood of $p$ in $S$. Then there is a $\nu<\omega_{1}$ such that $G \supset U_{\nu} \cap S$. But obviously $U_{\nu} \cap S=\left\{p_{\mu}: \nu<\mu<\omega_{1}\right\}$, hence $|S \backslash G| \leqslant\left|S \backslash U_{\nu}\right| \leqslant \omega$, i.e. any open cover of $S$ contains a member, whose complement is countable; consequently $S$ must be Lindelöf.

Proof of TheOrem 1. Now let $X$ be an uncountable compact space, and let $A \subset X,|A|=\omega_{1}$. If $|\bar{A}|=\omega_{1}$, too, we are done. Hence we assume $|\bar{A}|>\omega_{1}$.

If $p \in \bar{A}$ and $\chi(p, \bar{A})=\omega$, then we can choose a sequence from $A$ converging to $p$, hence

$$
|\{p \in \bar{A}: \chi(p, \bar{A}) \leqslant \omega\}| \leqslant \omega_{1}^{\omega}=\omega_{1} \quad(\text { by } \mathrm{CH}) .
$$

Applying the lemma, we see that if $\chi(p, \bar{A})=\omega_{1}$, for some $p \in \bar{A}$, then we are again done, hence we might assume $\chi(p, \bar{A}) \neq \omega_{1}$ for all $p \in \bar{A}$. Consequently if $p \in \bar{A}$ is such that it has a neighbourhood $U$ (in $\bar{A}$ ) with $|U| \leqslant \omega_{1}$, then we have $\psi(p, \bar{A})=\chi(p, \bar{A}) \leqslant \omega_{1}$, hence $\chi(p, \bar{A}) \leqslant \omega$.

Now let $G$ be the union of all open subsets of $\bar{A}$ of cardinality $\leqslant \omega_{1}$. Then from what we proved above, for every point $p$ of $G$ we have $\chi(p, \bar{A}) \leqslant \omega$, hence $|G| \leqslant \omega_{1}$. By the definition of $G, \bar{A} \backslash G$ is compact and has no isolated 
points (i.e. $\chi(p, \bar{A} \backslash G) \geqslant \omega$ for all $p \in \bar{A} \backslash G)$. By Proposition 2, there is a set $C \subset \bar{A} \backslash G$ with $|C|=\omega$ and $|\bar{C}| \geqslant \omega_{1}$. Again, we might assume that $|\bar{C}|$ $>\omega_{1}$.

Since $\bar{C}$ has a countable dense subset, we have $w(\bar{C}) \leqslant 2^{\omega}=\omega_{1}$; consequently $\chi(p, \bar{C}) \leqslant \omega_{1}$, for all $p \in \bar{C}$.

Moreover in the same way as above it follows that

$$
|\{p \in \bar{C}: \chi(p, \bar{C}) \leqslant \omega\}| \leqslant \omega_{1} .
$$

Hence there is a $p \in \bar{C}$ with $\chi(p, \bar{C})=\omega_{1}$, and thus, by the lemma, there is a Lindelöf subspace of cardinality $\omega_{1}$.

REMARK. A similar argument can be used to show that, assuming $\mathrm{GCH}$, for any $\kappa$ and any compact space of cardinality $>\kappa$ there is a $\kappa$-Lindelöf subspace of cardinality $\kappa^{+}$. It should be interesting to decide whether the first $\kappa$ in the conclusion could be lowered (perhaps even to $\omega$ ).

It is natural to raise the question why we have not asked about the existence of compact (i.e. closed) subspaces of cardinality $\omega_{1}$ of uncountable compact spaces. The space $\beta N$, however, shows that the answer to this question is negative as it is well known that any infinite closed subspace of $\beta N$ has the cardinality $2^{2^{\omega}}$.

Our next result shows that compact spaces without small infinite closed subsets must be large; under $\mathrm{CH}$ they have to have the same cardinality as $\beta N$.

Theorem 2. Suppose $X$ is compact and every infinite closed subset of $X$ is uncountable. Then $|X| \geqslant 2^{\omega_{1}}$.

Proof. Let $C$ be the set of all nonisolated points of $X$. First we claim that no point of $C$ is isolated in $C$, either. Indeed, let $p \in C$ and assume, on the contrary, that $U$ is a closed neighbourhood of $p$ in $X$ such that $U \cap C$ $=\{p\}$. However then $U$ is infinite, because $p$ is not isolated in $X$, and for any countably infinite $A \subset U$ the set $A \cup\{p\}$ is closed because its complement in $U$ consists of isolated points only. Thus $C$ must be dense in itself.

However, for no point $p \in C$ can we have $\chi(p, C)=\omega$, because in this case we could select from $C \backslash\{p\}$ an ordinary sequence $\left\langle p_{n}: n \in \omega\right\rangle$ of different points converging to $p$, and then $\left\{p_{n}: n \in \omega\right\} \cup\{p\}$ would be a countably infinite closed subset of $C$, and thus of $X$. But then for each $p \in C$ we have $\chi(p, C) \geqslant \omega_{1}$, consequently, by the Cech-Pospišil theorem, $|X| \geqslant$ $|C| \geqslant 2^{\omega_{1}}$.

\section{REFERENCES}

1. I. Juhász, Cardinal functions in topology, Math. Centre Tracts, no. 34, Mathematisch Centrum, Amsterdam, 1971. MR 49 \#478.

2. R. Engelking, Outline of general topology, Biblioteka Mat., Tom 25, PWN, Warsaw, 1965; English transl., North-Holland, Amsterdam; PWN, Warsaw; Interscience, New York, 1968. MR 36 \#4508; 37 \# 5836.

MTA Matematikai Kutató Intézete, H-1053 Budapest, Reáltanoda U. 13-15, Budapest, HUNGARY

EÖtovös Loránd Tudományegyetem, H-1088 Budapest, Múseum Krt. 6-8, Budapest, HUNGARY 